\title{
Análise de iniciativas envolvendo Lógica de Programação para alunos de Ensino Médio
}

\author{
Natália Bernardo Nunes ${ }^{1}$, Anelise Lemke Kologeski ${ }^{1}$ \\ ${ }^{1}$ Instituto Federal de Educação, Ciência e Tecnologia do Rio Grande do Sul (IFRS) - \\ Campus Osório
}

\author{
Rua Santos Dumont, 2127 - Albatroz, Osório - RS, 95520-000 \\ nataliabernunes@gmail.com, anelise.kologeski@osorio.ifrs.edu.br
}

\begin{abstract}
Technology's influence on our society increases everyday. However, it has been superficially observed in most Brazilian secondary schools, causing negative impacts on educational rates. An alternative to soften this situation can be through the inclusion of the Logic of Programming in the school. To investigate initiatives in this regard, a search has been done for annals of events that address educational practices related to the use of Programming Logic, analyzing their results. So, 187 articles have been found related to the topic, of which 38 were applied for secondary school, being 18 of these with statistics for analysis. The results of the initiatives vary between $48.7 \%$ and $90.9 \%$ of improvement, motivating the expansion of research in this area.
\end{abstract}

Resumo. A influência da tecnologia em nossa sociedade cresce a cada dia. No entanto, ela tem sido observada superficialmente na maioria das escolas de Ensino Médio brasileiras, causando impactos negativos para os índices educacionais. Uma alternativa para amenizar esta situação pode se dar através da inclusão da Lógica de Programação na escola. Para investigar iniciativas neste aspecto, realizou-se uma busca por anais de eventos que abordam práticas educativas relacionadas ao uso da Lógica de Programação, analisando seus resultados. Então, 187 artigos foram encontrados relacionados ao tema, dos quais 38 foram aplicados ao Ensino Médio, sendo 18 destes com estatísticas para análise. Os resultados das iniciativas variam entre 48,7\% e 90,9\% de aproveitamento, motivando a ampliação da pesquisa nesta área.

\section{Introdução}

Muitos hábitos do dia a dia estão se tornando cada vez mais relacionados de alguma forma ao uso de dispositivos eletrônicos e recursos digitais, tais como sistemas operacionais, aplicativos, telas interativas, jogos eletrônicos e Internet, tornando a tecnologia um assunto de interesse para a maioria da pessoas. Direcionando esta afirmação para os jovens, ela torna-se ainda mais contextualizada, já que este público considera as novas tecnologias uma realidade irretornável na vida contemporânea (Sousa, 2011), proporcionando as mais diversas experiências aos nativos digitais, que são tanto os estudantes de escolas públicas quanto privadas.

Apesar dos grandes avanços da tecnologia, possibilitando o alcance cada vez maior de usuários, um problema recorrente na sociedade atual são os baixos índices de 
avaliação da educação em todo o país. Segundo o site Agenda $2020^{1}$, uma das causas da falta de desenvolvimento educacional no estado do Rio Grande do Sul é a "Inadequação da educação escolar às exigências do século XXI", apontando que há poucos indícios de práticas inovadoras, em especial no Ensino Médio. Essa ausência ocorre justamente no período em que deveria haver uma conexão entre o currículo escolar e as demandas do mercado de trabalho, o que dificilmente ocorre nas escolas do estado. Um dos fatores analisados nesta plataforma é o chamado Índice de Desenvolvimento da Educação Básica (IDEB) ${ }^{2}$, que consiste em uma prova realizada para mensurar o aprendizado dos alunos em conteúdos relacionados à leitura e à resolução de problema. Nas últimas 3 edições, os resultados mostraram que o desempenho dos estudantes foi menor que o esperado, nas escolas públicas de Ensino Médio de todos os municípios da região do Litoral Norte do Rio Grande do Sul. Os resultados nacionais do IDEB, para as 3 últimas edições, podem ser visualizados na tabela 1 , considerando 10 como a nota máxima.

Tabela 1. IDEB atingido e esperado das 3 últimas edições para o Ensino Médio no Brasil. IDEB, 2019

\begin{tabular}{|c|c|c|c|c|c|c|}
\hline Rede & $\begin{array}{c}2013 \\
\text { Atingido }\end{array}$ & $\begin{array}{c}2013 \\
\text { Esperado }\end{array}$ & $\begin{array}{c}2015 \\
\text { Atingido }\end{array}$ & $\begin{array}{c}2015 \\
\text { Esperado }\end{array}$ & $\begin{array}{c}2017 \\
\text { Atingido }\end{array}$ & $\begin{array}{c}2017 \\
\text { Esperado }\end{array}$ \\
\hline Privada & 5,4 & 6,0 & 5,3 & 6,3 & 5,8 & 6,7 \\
\hline Pública & 3,4 & 3,6 & 3,5 & 4,0 & 3,5 & 4,4 \\
\hline
\end{tabular}

Embora as médias esperadas estejam aumentando ao longo das edições, essa evolução não é vista de maneira concreta quando observamos as médias atingidas. De acordo com a plataforma Agenda $2030^{3}$, para assegurar a educação inclusiva e equitativa de qualidade até o ano de 2030, é necessário aumentar substancialmente o número de jovens e adultos que tenham habilidades relevantes, inclusive competências técnicas e profissionais, para emprego, trabalho decente e empreendedorismo. Logo, os alunos deveriam estar mais preparados do que realmente estão, fazendo com que esta seja uma problemática muito recorrente em todas as escolas brasileiras e sendo necessária a investigação de uma solução eficiente para amenizar este problema.

Uma pesquisa do Instituto Federal da Paraíba - IFPB (Câmara, 2016) aponta que alunos de Ensino Fundamental e médio que possuem contato com o ensino de programação acabam desenvolvendo capacidade e competência para a resolução de problemas e para o raciocínio lógico. Logo, um possível caminho para contornar a presente situação, poderia ser a implementação de ações que desenvolvam o pensamento computacional e o raciocínio lógico nas escolas, junto aos estudantes.

Por este motivo, este trabalho visa investigar o impacto que o ensino da lógica de programação, desde cedo, pode trazer para a vida dos estudantes, tanto da perspectiva dos autores de propostas quanto dos alunos envolvidos, avaliando a consequência disso no desempenho escolar destes alunos. Contudo, normalmente são encontradas diversas iniciativas voltadas para o Ensino Fundamental. Como diferencial, este trabalho pretende mostrar uma revisão sistemática, realizada em busca de projetos

\footnotetext{
${ }^{1}$ https://agenda2020.com.br/

${ }^{2}$ http://ideb.inep.gov.br/

${ }^{3}$ https://agenda2030.com.br/
} 
VIII Congresso Brasileiro de Informática na Educação (CBIE 2019)

Anais dos Workshops do VIII Congresso Brasileiro de Informática na Educação (WCBIE 2019)

que promovem atividades auxiliando os alunos a desenvolverem a lógica de programação, especificamente em escolas de Ensino Médio.

\section{Objetivos e justificativa}

Existem muitas iniciativas em todo o Brasil que contribuem para o Ensino Médio através da lógica de programação. Logo, esta pesquisa visa avaliar os resultados obtidos nestes projetos, investigando as propostas encontradas, analisando se esta vivência é válida para o desenvolvimento do raciocínio lógico dos alunos, e se esta seria uma alternativa viável para a transformação social. Logo, o objetivo da análise é investigar se iniciativas voltadas à Lógica de Programação possuem impacto no desenvolvimento educacional dos alunos participantes, quais atividades são realizadas e qual o seu nível de aproveitamento, bem como as regiões do Brasil em que são implantadas e indicadores educacionais destas regiões.

Neste contexto, almeja-se auxiliar estudantes e docentes a incentivarem a inclusão digital em suas instituições de ensino por meio deste levantamento de dados, buscando identificar quais são as iniciativas mais eficientes, bem como onde e quando aplicá-las, compilando os dados obtidos neste estudo.

Já é possível encontrar resultados positivos em projetos voltados para Educação Básica, como por exemplo o projeto "Logicando" (Kologeski, 2016), da universidade Feevale, em Novo Hamburgo/RS, que apresenta até $45 \%$ de melhoria no desenvolvimento dos alunos, utilizando plataformas que desenvolvem o pensamento computacional. No Instituto Federal do Rio Grande do Sul - Campus Osório, também é desenvolvido um projeto similar, para o mesmo público-alvo (séries finais do Ensino Fundamental), chamado de "Programando Fácil: Conhecendo a Computação" (Fabrício, 2018), onde os autores citam o projeto como uma forma simples de contribuição à Educação Básica, com melhorias de até $40 \%$ no desenvolvimento dos alunos, utilizando uma plataforma digital específica e gratuita. Contudo, o foco da pesquisa deste trabalho são projetos semelhantes, mas que tenham como público alvo alunos de Ensino Médio, visando traçar um novo caminho não apenas na educação, mas também na formação destes estudantes mediante a sociedade, preparando-os adequadamente para o mercado trabalho, além de investigar se há uma contribuição favorável para a transformação na vida daqueles que participam de atividades educacionais ligadas à tecnologia.

\section{Metodologia}

Para que ocorra o desenvolvimento deste trabalho, determinou-se que seriam investigados os anais de eventos pertinentes, como o workshop do Congresso Brasileiro de Informática na Educação - $\mathrm{CBIE}^{4}$ e o Simpósio Brasileiro de Informática na Educação - SBIE5 , bem como publicações da revista Renote - Novas Tecnologias na Educação da Universidade Federal do Rio Grande do $\mathrm{Sul}^{6}$, dando ênfase às últimas edições (de 2015 a 2018).

\footnotetext{
${ }^{4}$ https://www.br-ie.org/pub/index.php/wcbie

${ }^{5}$ https://www.br-ie.org/pub/index.php/sbie

${ }^{6}$ https://seer.ufrgs.br/renote
} 
VIII Congresso Brasileiro de Informática na Educação (CBIE 2019)

Anais dos Workshops do VIII Congresso Brasileiro de Informática na Educação (WCBIE 2019)

Primeiramente, realizou-se uma seleção de artigos com as palavras-chave "lógica de programação", "pensamento computacional" e "raciocínio lógico" em seu título e/ou resumo. Em seguida, refinou-se a pesquisa em busca apenas daqueles trabalhos destinados ao Ensino Médio, tendo em vista que muitos dos projetos encontrados eram destinados ao Ensino Fundamental ou iniciativas envolvendo os alunos dos primeiros semestres de cursos superiores voltados à área da tecnologia. Para fins de comparação, foram considerados também projetos que continham estatísticas sobre o aproveitamento do projeto, permitindo a realização de uma comparação posterior. A procura por depoimentos dos participantes das iniciativas também foi de extrema importância, tendo em vista que apresentam a real opinião dos alunos sobre o tema e qual o real impacto daquele projeto em suas vidas.

\section{Resultados}

A quantidade total de projetos encontrados, de acordo com as palavras-chave utilizadas, está listada na tabela 2:

Tabela 2. Relação dos projetos encontrados nos anais dos workshops do CBIE, SBIE e revista Renote de 2015 a 2018. Autoria Própria.

\begin{tabular}{|c|c|c|c|}
\hline Palavra-chave & $\begin{array}{c}\text { Projetos } \\
\text { Encontrados }\end{array}$ & $\begin{array}{c}\text { Publico-alvo: } \\
\text { Ensino Médio }\end{array}$ & $\begin{array}{c}\text { Projetos com } \\
\text { Estatísticas }\end{array}$ \\
\hline $\begin{array}{c}\text { Pensamento } \\
\text { Computacional }\end{array}$ & 99 & 23 & 9 \\
\hline Raciocínio Lógico & 37 & 7 & 4 \\
\hline $\begin{array}{c}\text { Lógica de } \\
\text { Programação }\end{array}$ & 53 & 11 & 7 \\
\hline
\end{tabular}

Com base na tabela 2 é possível perceber que uma minoria dos trabalhos é voltada ao Ensino Médio, levantando a hipótese de que os pesquisadores da atualidade direcionam a inclusão digital para o Ensino Fundamental, alinhando-a com a formação da maturidade e até mesmo a alfabetização dos indivíduos, auxiliando na formação do que conhecemos hoje como os nativos digitais, ou para o Ensino Superior, considerando estudantes que já possuem o contato com a Lógica de Programação nos cursos que realizam, servindo apenas como um auxílio nas disciplinas da graduação. Isso mostra que ainda há muito para ser explorado, justamente em uma faixa etária onde os estudantes estão se preparando e se qualificando para ingresso no mercado de trabalho. Além disso, houve uma dificuldade na investigação dos projetos encontrados, devido à falta de estatísticas em seus resultados que relacionassem de maneira explícita o impacto causado, impossibilitando dados concretos para fins de comparação. Os resultados das estatísticas referentes ao desempenho dos projetos variam entre $48,7 \%$ e 90,9\% referentes ao aumento das notas dos participantes antes e depois das iniciativas, bem como satisfação, interesse dos participantes, dentre outros fatores. Apenas 6

\footnotetext{
${ }^{7}$ Ao contabilizar, foram 189 artigos, dos quais 41 eram de Ensino Médio e 20 deles possuíam estatísticas. No entanto, alguns trabalhos apareceram duas vezes com a mesma palavra-chave ou em dois eventos diferentes, havendo uma continuidade nos seus resultados. Contabilizando apenas uma vez cada uma dessas publicações, totalizam-se 187 artigos, 38 de Ensino Médio e 18 deles com estatísticas.
} 
VIII Congresso Brasileiro de Informática na Educação (CBIE 2019)

Anais dos Workshops do VIII Congresso Brasileiro de Informática na Educação (WCBIE 2019)

trabalhos possuíam depoimentos feitos pelos próprios participantes, e esta falta na grande maioria dos artigos também sugerem um novo tipo de análise em busca da real opinião dos alunos sobre a contribuição que a Lógica de Programação implica em seus cotidianos e suas formações acadêmicas.

\subsection{Projetos analisados dos workshops do CBIE}

- Jogos de Programar como uma Abordagem para os Primeiros Contatos dos Estudantes com a Programação (Zanchett, 2015): Este projeto contou com 14 estudantes, sendo que apenas um possuía contato com programação antes da iniciativa. No final, os autores obtiveram $85,7 \%$ de aproveitamento, ou seja, esta é a porcentagem de alunos que concluíram o projeto compreendendo a programação.

- Um relato sobre estratégias de motivação e ensino de lógica de programação para e por mulheres (Sass, 2018): Este projeto contou com 26 estudantes, todas mulheres, pois o principal objetivo era motivar as alunas de Ensino Médio a ingressarem na Informática. Além de dados relacionados à motivação das mulheres na área, a pesquisa também traz dados referentes ao desenvolvimento delas ao longo das aulas. Diante de um teste final, as alunas acertaram, em média, $88 \%$ das questões.

- Uma Experiência Interdisciplinar no Ensino de Algoritmos e Matemática em um Contexto Binacional (Cordenonzi, 2015): Este projeto contou com 28 estudantes. Trata-se de um software desenvolvido pelos autores para auxiliar os estudantes na resolução de problemas. Eram aplicados testes aos participantes e $87 \%$ dos mesmos consideraram que, ao cometer um erro, o software ajudava-os a entendê-lo.

- Explorando o Uso da Robótica na Educação Básica: um estudo sobre ações práticas que estimulam o Pensamento Computacional (Souza, 2018): Este projeto contou com 41 estudantes de duas escolas diferentes, sendo que 39 nunca possuíram contato com robótica anteriormente. Foram realizadas atividades com os estudantes e, no final, as duas escolas juntas obtiveram uma melhoria de $48,7 \%$ no desempenho dos alunos participantes do projeto.

- Prática de ensino de Programação de Computadores com Robótica Pedagógica e aplicação de Pensamento Computacional (Zanetti, 2015): Este projeto contou com 6 estudantes do Curso Técnico em Informática Integrado ao Ensino Médio, onde foram apresentados novos métodos de desenvolver o Pensamento Computacional. Um total de $50 \%$ dos alunos acharam o método mais motivador e mais claro que o tradicional, e $67 \%$ dos alunos conseguiram, ao final do projeto, solucionar as atividades sem problemas. Além disso, $83 \%$ dos alunos conseguiram identificar totalmente os seus erros (quando cometidos) e acreditando que o método ajuda totalmente na disciplina de programação.

- "Hello World": relato de experiência de um curso de iniciação à programação (Ferreira, 2016): Este projeto contou com 43 estudantes, e após o curso foi aplicado um questionário. De todos os participantes, 29 responderam às questões propostas. No final, 86,21\% revelaram que as expectativas no curso foram atendidas.

- Experiência no Programa Institucional de Bolsas de Iniciação à Docência (PIBID): Desenvolvimento do Raciocínio Lógico e Algoritmo na Educação Básica (Lima, 2015): Este projeto contou com 21 estudantes. No pré teste, a avaliação foi respondida por 19 alunos, onde 17 ficaram acima da média estipulada na parte de Língua Portuguesa e Matemática, porém apenas 6 ficaram acima da média na parte de Lógica. Após o curso de iniciação à Programação, foi realizado um pós teste por 17 participantes, onde 13 ficaram acima da média em Língua Portuguesa, 14 em 
VIII Congresso Brasileiro de Informática na Educação (CBIE 2019)

Anais dos Workshops do VIII Congresso Brasileiro de Informática na Educação (WCBIE 2019)

Lógica e 17 em Matemática, observando um nítido destaque entre o pré teste e o pós teste. Por fim, foi proposto um teste através de um algoritmo, onde 59\% obtiveram notas acima da média.

\subsection{Projetos analisados do SBIE}

- Ensino de Lógica de Programação no Ensino Médio e suas aplicações na Neurociência (Bastos, 2015): Esta pesquisa analisa o desenvolvimento dos alunos através de possíveis mudanças na fisiologia cerebral após o desenvolvimento da Lógica Computacional. Foram realizadas 2 análises: uma pré e outra pós este treinamento em alunos dos 2 últimos anos do Ensino Médio. Ao analisar o cérebro dos indivíduos no pós treinamento, um número significativo de áreas no cérebro foram ativadas em relação ao pré treinamento. Além disso, a pesquisa visa fomentar uma revisão na formação docente, para que o ensino básico saia beneficiado com o ensino da Lógica de Programação.

- Algo+ Uma ferramenta para o apoio ao ensino de Algoritmos e Programação para alunos iniciantes (Amaral, 2017): Este projeto, apesar de ter sido aplicado em alunos de Ensino Superior, também pode auxiliar alunos de Ensino Médio já que ensina a Lógica de Programação para iniciantes, desenvolvendo outras habilidades. Contou com a participação de 47 alunos e no final um questionário foi realizado com 36 deles, com questões referentes ao grau de aproveitamento obtido. Mais de $75 \%$ dos alunos responderam de forma positivamente, tornando o projeto produtivo.

- Tri-Logic - Proposta Lúdica Gamificada para o Ensino e Aprendizagem da Lógica de Programação com o Uso da Mineração de Dados como Ferramenta de Auxílio ao Professor (Barbosa, 2017): Trabalha com frameworks para gamificação que foram testados com 20 alunos do Curso Técnico em Desenvolvimento de Sistemas de Integrado ao Ensino Médio. Após a aplicação da atividade, 90,9\% dos alunos responderam um questionário dizendo-se motivados a seguirem aprendendo Lógica de Programação, apontando um aproveitamento positivo.

- Construção de ambiente de ensino de robótica remota: democratizando o desenvolvimento do pensamento computacional em alunos da Educação Básica (Costella, 2017): Esta iniciativa desenvolve o Pensamento Computacional por meio da Robótica e plataformas com blocos. Com 47 usuários cadastrados, o projeto obteve uma média de 4,7 execuções por usuário. A plataforma desenvolvida conta com um questionário, onde $64,3 \%$ das respostas apontaram o grau máximo de satisfação, e 57,1\% utilizariam a plataforma novamente se houvessem mais desafios.

- Explorando Robótica com Pensamento Computacional no Ensino Médio: Um estudo sobre seus efeitos na educação (Souza, 2016): Propõe oficinas de robótica de uma maneira lúdica e compreensível. O projeto foi aplicado com 729 alunos do primeiro ano do Ensino Médio, e comprovou que o Pensamento Computacional melhora o desempenho dos alunos em oficinas de robótica, e consequentemente em disciplinas do quadro escolar, como Matemática e Língua Portuguesa.

- Pensamento Computacional e Robótica: Um Estudo Sobre Habilidades Desenvolvidas em Oficina de Robótica Educacional (Oliveira, 2016): O trabalho faz um análise do impacto que o Pensamento Computacional pode causar em uma oficina de robótica. Um total de 18 alunos participaram de pré e pós testes. Os resultados apresentaram uma melhoria não apenas com foco no erro e no acerto das questões, mas também na organização das respostas. Dos 18 alunos envolvidos, 12 
VIII Congresso Brasileiro de Informática na Educação (CBIE 2019)

Anais dos Workshops do VIII Congresso Brasileiro de Informática na Educação (WCBIE 2019)

deles responderam corretamente a questão final do pós teste, que tratava de todas as habilidades trabalhadas durante a oficina.

- Programando para criar objetos de aprendizagem digitais de "Ondulatória" (Franzoia, 2018): Para esta iniciativa, foi proposto aos alunos do $2^{\circ}$ ano do Ensino Médio que desenvolvessem o Pensamento Computacional para criar objetos digitais de aprendizagem com ênfase em Ondulatória. Participaram da proposta 42 discentes, havendo uma evolução significativa para a maioria dos estudantes.

- T-mind: um Aplicativo Gamificado para Estímulo ao Desenvolvimento de Habilidades do Pensamento Computacional (Pessoa, 2017): Este projeto foi contabilizado duas vezes nos resultados, por ter sido encontrado nas buscas por "Pensamento Computacional" e "Raciocínio Lógico". No entanto, não apresentou resultados distintos em suas duas publicações, por isso é descrito apenas uma vez. Trata-se de um aplicativo de aprendizagem que não requer conhecimento prévio, aplicado em 25 alunos de Ensino Médio, sendo que 57\% avaliaram os recursos gamificados como muito estimulantes.

- Uma análise de um jogo educacional sob a ótica do Pensamento Computacional (Junior, 2018): Apresenta a aplicação e avaliação de um jogo computacional, aplicado com 16 alunos. Foi realizado um pré e um pós teste com conhecimentos de Pensamento Computacional, em três grupos diferentes. Todos obtiveram melhorias, significativas, segundo os autores.

\subsection{Projetos analisados da revista Renote}

- Arcabouço Pedagógico combinado com o glossário técnico de computação em LIBRAS: uma experiência com Lego Mindstorms NXT (Granada, 2018): Esta iniciativa elaborou um glossário de sinais em LIBRAS com termos técnicos, estimulando o raciocínio lógico para alunos surdos. A ação contou com 4 alunos surdos de uma escola de Ensino Médio bilíngue. Cada aluno foi avaliado de forma individual, analisando quatro categorias: conhecimento, compreensão, atenção e interação. Os autores consideram esta iniciativa uma oportunidade de acesso à computação para os estudantes surdos.

- Tri-Logic: Um Ambiente Gamificado como Ferramenta de Auxílio ao ensino de aprendizagem de Lógica de Programação (Natal, 2018): Esta iniciativa já havia sido encontrada nos anais do SBIE, no entanto, foi publicada novamente na revista Renote, com resultados atualizados. O projeto visa motivar alunos do curso técnico em Desenvolvimento de Sistemas Integrado ao Ensino Médio a aprenderem os conteúdos relacionados à Lógica de Programação, em um ambiente que conta com o auxílio de um software desenvolvido pelos próprios autores. Dos participantes que utilizaram o sistema, 73,3\% acharam a sua utilização divertida, 53,3\% afirmaram que este fator os motivou a continuar, $60 \%$ se sentiram confiantes em realizar um teste com os conteúdos tratados no ambiente e $88,7 \%$ sentiram-se motivados a aprender Lógica de Programação. Além dos novos resultados, foram apresentados comentários feitos pelos participantes, que sugeriram novas fases com maior grau de dificuldade.

Tendo em vista os artigos encontrados, observou-se que 38,9\% das iniciativas trabalham com softwares lúdicos, $27,8 \%$ são oficinas de programação e $33,3 \%$ são oficinas de robótica. 
A predominância de projetos na região Sul do Brasil $(42,1 \%)$ também foi observada, bem como a ausência na região Centro-Oeste dentre os projetos analisados. Com estes dados, foi realizada uma nova análise com o auxílio da plataforma do IDEB, comparando-se os índices destas duas regiões, onde observou-se que existe uma expectativa para o ano 2019 de 0,4 pontos a mais na região Sul em relação à região Centro-Oeste para as escolas de Ensino Médio. Referindo-se apenas às escolas públicas destas regiões, a diferença torna-se ainda maior: espera-se 0,6 pontos a mais na região Sul, apontando uma possível relação com as iniciativas relacionadas à Lógica de Programação em escolas dessa região, já que existem mais iniciativas na região Sul enquanto tem-se a ausência de propostas neste contexto para a região Centro-Oeste.

\section{Conclusão}

Com base nos resultados dos 38 trabalhos encontrados após a busca inicial, foi possível observar um resultado satisfatório em relação ao desenvolvimento dos alunos com o auxílio da Lógica de Programação, diante de diversos indicadores e depoimentos observados, o que é motivador para seguir investigando essa temática, e principalmente se é uma alternativa viável para o desenvolvimento dos alunos do Ensino Médio.

Futuramente, pretende-se buscar por dados de outros eventos realizados no Brasil relacionados ao Pensamento Computacional, ao Raciocínio Lógico e a Lógica de Programação na Educação, como por exemplo, a revista Observatório ${ }^{8}$, além de eventos internacionais, a fim de observar-se como ocorrem iniciativas semelhantes em diferentes países. Além disso, uma pesquisa de campo será efetuada, para realizar a busca por depoimentos, com questionários anônimos, abordando a real opinião dos alunos sobre o uso da Lógica de Programação e respectivo o impacto causado em suas vidas diante deste contato, iniciando pelo município de Osório/RS, onde a pesquisa está sendo realizada, e estendendo-se para outros municípios da região do Litoral Norte do Rio Grande do Sul. Assim, objetiva-se identificar se os alunos de Ensino Médio da região considerada já tiveram de fato oportunidades semelhantes aos trabalhos encontrados, uma vez que observou-se de forma muito breve a real opinião e a escassez de comentários dos alunos nos trabalhos investigados.

\section{Referências}

Amaral, E., Camargo, A., Gomes, M., Richa, C., Becker, L. ALGO+ Uma ferramenta para o apoio ao ensino de Algoritmos e Programação para alunos iniciantes. Anais do XXVIII Simpósio Brasileiro de Informática na Educação. Recife, PE, Brasil, 2017.

Barbosa, B., Much, B. S., Silva, C. O. B., Bigolin, M., Silva, S. J. R. Tri-Logic Proposta Lúdica Gamificada para o Ensino e Aprendizagem da Lógica de Programação com o Uso da Mineração de Dados como Ferramenta de Auxílio ao Professor. XXVIII Simpósio Brasileiro de Informática na Educação. Recife, PE, Brasil, 2017.

\footnotetext{
${ }^{8}$ https://sistemas.uft.edu.br/periodicos/index.php/observatorio/index
} 
VIII Congresso Brasileiro de Informática na Educação (CBIE 2019)

Anais dos Workshops do VIII Congresso Brasileiro de Informática na Educação (WCBIE 2019)

Bastos, N. S., Adamatti, D. F., Carvalho, F. A. H. Ensino de Lógica de Programação no Ensino Médio e suas implicações na Neurociências. XXVI Simpósio Brasileiro de Informática na Educação. Maceió, AL, Brasil, 2015.

Câmara, F. S. S., Silva, M. A. P, Silva, R. M. B. A Lógica de Programação como ferramenta para o ensino e a aprendizagem da Matemática. Anais do II Congresso Internacional de Educação Inclusiva, Campina Grande, PB, Brasil, 2016.

Cordenonzi, W., Cardoso, V., Luz, H., Amaral, E. Uma Experiência Interdisciplinar no Ensino de Algoritmos e Matemática em um Contexto Binacional. Anais do IV Congresso Brasileiro de Informática na Educação, Maceió, AL, Brasil, 2015.

Costella, L., Trentin, M. A. S., Amarante, V. G., Teixeira, A. C. Construção de ambiente de ensino de robótica remota: democratizando o desenvolvimento do pensamento computacional em alunos da Educação Básica. Anais do XXVIII Simpósio Brasileiro de Informática na Educação. Recife, PE, Brasil, 2017.

Fabrício, V. S., Nunes, N. B., Julio, M. B., Espíndola, R. W. P., Kologeski, A. L. Programando Fácil: Conhecendo a Computação. Anais do XI Congresso Sul Brasileiro de Computação, Criciúma, SC, Brasil, 2018.

Ferreira, A. C., Santos, J., Silva, R., Oliveira, A. T., Zabot, D., Santos, D. A., Matos, E. S. "Hello World": relato de experiência de um curso de iniciação à programação. Anais do V Congresso Brasileiro de Informática na Educação, Uberlândia, MG, Brasil, 2016.

Franzoia, F. H., Pires, F. G. S., Teixeira, K. B., Lima, P. A. Programando para criar objetos de aprendizagem digitais de "Ondulatória". Anais do XXIX Simpósio Brasileiro de Informática na Educação. Fortaleza, CE, Brasil, 2018.

Granada, R. P., Botelho, S. S. C., Barwaldt, R. Arabouço Pedagógico combinado com o glossário técnico de computação em LIBRAS: uma experiência com Lego Mindstorms NXT. In: Renote, 2018. Revista Renote, novas tecnologias na educação. V. $16, \quad \mathrm{~N}^{\circ} 2$ 2018. Disponível em: <https://seer.ufrgs.br/renote/article/view/89278/51518 >. Acesso em: 27 jul. 2019.

Júnior, B. A. S., Cavalheiro, S. A. C., Foss. L. Uma análise de um jogo educacional sob a ótica do Pensamento Computacional. Anais do XXIX Simpósio Brasileiro de Informática na Educação. Fortaleza, CE, Brasil, 2018.

Kologeski, A. L., Silva, C. G., Barbosa, D. N. F., Mattos, R. R., Miorelli, S. T. Desenvolvendo o Raciocínio Lógico e o Pensamento Computacional: experiências no contexto do projeto Logicando. Revista Renote, novas tecnologias na educação, Porto Alegre, RS, Brasil, 2016.

Lima, A. C., Sousa, D. F. Experiência no Programa Institucional de Bolsas de Iniciação à Docência (PIBID): Desenvolvimento do Raciocínio Lógico e Algoritmo na Educação Básica. Anais do IV Congresso Brasileiro de Informática na Educação, Maceió, AL, Brasil, 2015.

Natal, M. E. C. et. al. Tri-Logic: Um Ambiente Gamificado como Ferramenta de Auxílio ao ensino de aprendizagem de Lógica de Programação. In: Renote, 2019. Revista Renote, novas tecnologias na educação. V. 16, $\mathrm{N}^{\mathrm{o}}$ 2, 2018. Disponível em: $<$ https://seer.ufrgs.br/renote/article/view/89298/51536>. Acesso em: 27 jul. 2019. 
VIII Congresso Brasileiro de Informática na Educação (CBIE 2019)

Anais dos Workshops do VIII Congresso Brasileiro de Informática na Educação (WCBIE 2019)

Oliveira, E. J. S., Araújo, A. L. S. O., Pensamento Computacional e Robótica: Um Estudo Sobre Habilidades Desenvolvidas em Oficina de Robótica Educacional. Anais do XXVII Simpósio Brasileiro de Informática na Educação. Uberlândia, MG, Brasil, 2016.

Pessoa, F. I. R., Araújo, A. L. S. O., Andrade, W. L., Guerrero, D. D. S. T-mind: um Aplicativo Gamificado para Estímulo ao Desenvolvimento de Habilidades do Pensamento Computacional. Anais do XXVIII Simpósio Brasileiro de Informática na Educação. Recife, PE, Brasil, 2017.

Sass, C., Kanashiro, C., Yahata, E., Nocete, G., Baldi, J., Tenore, L., Santana, N., Ribeiro, T., Rodriguez, C., Sato, C., Goya, D., Venero, M., Rocha, R. V. Um relato sobre estratégias de motivação e ensino de lógica de programação para e por mulheres. Anais do VII Congresso Brasileiro de Informática na Educação. Fortaleza, CE, Brasil, 2018.

Sousa, C. A. M. Novas linguagens e sociabilidades: como uma juventude vê novas tecnologias. In: Interacções, 2011. Revista Interacções, Repositório Científico do Instituto Politécnico de Santarém. V. 7, No 17, 2011. Disponível em: $<$ https://repositorio.ipsantarem.pt/handle/10400.15/508>. Acesso em: set. 2019.

Souza, I. M. L., Sampaio, L. M. R., Andrade, W. L. Explorando o Uso da Robótica na Educação Básica: um estudo sobre ações práticas que estimulam o Pensamento Computacional. Anais do VII Congresso Brasileiro de Informática na Educação. Fortaleza, CE, Brasil, 2018.

Souza, I. M. L., Rodrigues, R. S., Andrade, W. L. Explorando Robótica com Pensamento Computacional no Ensino Médio: Um estudo sobre seus efeitos na educação. Anais do XXVII Simpósio Brasileiro de Informática na Educação. Uberlândia, MG, Brasil, 2016.

Walgprog, 2019. Workshop de Ensino em Pensamento Computacional, Algoritmos e Programação. Disponível em: <http://walgprog.gp.utfpr.edu.br/>. Acesso em: mai. 2019.

Zanchett, G. A., Vahldick, A., Raabe, A. Jogos de Programar como uma Abordagem para os Primeiros Contatos dos Estudantes com a Programação. Anais do IV Congresso Brasileiro de Informática na Educação, Maceió, AL, Brasil, 2015.

Zanetti, H. A. P., Oliveira, C. L. V. Prática de ensino de Programação de Computadores com Robótica Pedagógica e aplicação de Pensamento Computacional. Anais do IV Congresso Brasileiro de Informática na Educação, Maceió, AL, Brasil, 2015. 